\title{
Zum Redaktionswechsel
}

Es war sinnvoll, unsere Zeitschrift nach dem Renaissancegelehrten Conrad Geßner, der zugleich Arzt und Naturforscher war, zu benennen, denn ihre Entstehung und Entwicklung war dem Zusammenwirken von Ärzten und Naturforschern zu verdanken. Der Initiant, der Zoologe Jean Strohl, hat das Erscheinen der ersten Hefte nicht mehr erlebt, jedoch stammte der Einführungsartikel aus seiner Feder. Der Pharmakologe Hans Fischer, Mitinitiant und Freund Strohls, hat dann die Redaktion übernommen und bis weit über seine Emeritierung hinaus bis zum 30. Jahrgang geführt. Erwin H. Ackerknecht hat ihm in Gesnerus 30 (1976) 163, einen schönen Nachruf gewidmet. Auf die unvergeßliche Ära Fischer folgte die Redaktionszeit des Psychiaters Hans H. Walser 1974-1980. Seine Persönlichkeit ist anläßlich seines 60. Geburtstages in Gesnerus 37 (1980) 1 von Huldrych M. Koelbing gewürdigt worden. Walser hat es verstanden, in Fortsetzung des Werks von Fischer, Ansehen und Verbreitung der Zeitschrift weiter zu fördern. Er hat angestrebt, neben den drei Landessprachen auch Beiträge in spanischer und englischer Sprache abzudrucken. Es wurden systematisch englische Zusammenfassungen eingeführt. Walser hat sich die Mitarbeit von Heinz Balmer sichern können, der seit 1976 als Mitredaktor zeichnete und für die naturwissenschaftlichen Beiträge zuständig war. Balmer war den Lesern der Zeitschrift seit Jahren bekannt, als Ersteller des wertvollen Registers der ersten 25 Jahrgänge und als Verfasser einer Reihe von bemerkenswerten Artikeln zu geographischen und allgemein-naturwissenschaftlichen Themen. Er hat nach dem Rücktritt Walsers die Redaktion allein weitergeführt, unter Assistenz einer Redaktionskommission von drei Ärzten. Mit der gegenwärtigen Ablösung geht die Verantwortung wieder an einen Mediziner über. Seit der Ära Walser hat das Medizinhistorische Institut der Universität Zürich seine Dienste als Redaktionssekretariat zur Verfügung gestellt, und mit dem Redaktionswechsel übernimmt in verdankenswerter Weise nun die Historikerin Marie-Louise Portmann mit der Medizinhistorischen Bibliothek der Universität Basel diese Aufgabe.

Carl Haffter

Redaktionsschlüsse für die beiden Hefte des Gesnerus-Jahrgangs:

28. Februar für Heft 1/2, das anfangs Juni erscheint.

31. August für Heft 3/4, das anfangs Dezember erscheint. 
Gesnerus erscheint halbjährlich in 2 Heften. In außerordentlichen Fällen erscheinen auch drei Hefte. Preis des Heftes Fr.45.-, des Jahrganges im Abonnement Fr.72.-- Mitglieder der Schweizerischen Gesellschaft für Geschichte der Medizin und der Naturwissenschaften erhalten die Zeitschrift gratis (Mitgliederbeitrag Fr.55.-, von 65 J. an Fr.45.-, Assistenten und Studenten Fr. 35.-, Postscheck 80-25315). Bestellungen an den Verlag Sauerländer AG, Postfach, CH-5001 Aarau, Tel. 064/22 1264, Telex 981195 sag ch (Preisstand 1.1.1982, Preisänderungen vorbehalten).

Gesnerus paraît deux fois par an (extraordinairement trois fois). Prix du fascicule (double ou simple) frs. 45.-, abonnement annuel frs. 72.-. Les membres de la Société suisse d'histoire de la médecine et des sciences naturelles reçoivent la revue gratuitement (cotisation frs. 55.-, à part de 65 ans frs.45.-, assistants et étudiants frs.35.-, compte de chèques postaux 80-25315). Commandes à l'éditeur Sauerländer AG, case postale, CH-5001 Aarau, tél. 064/22 12 64, télex 981195 sag ch (prix valable le 1.1.1982, changements de prix réservés).

\section{Mitgliedschaft / Adhésions}

Anmeldung beim Sekretariat (Dr.med. Alfred W.Gubser, Medizinhistorisches Institut der Universität Zürich, Rämistraße 71, 8006 Zürich) oder bei einem anderen Vorstandsmitglied. Die Mitglieder erhalten die Veröffentlichungen der Gesellschaft zu einem Vorzugspreis. Die Mitglieder sind zur Teilnahme an allen Veranstaltungen der Gesellschaft berechtigt, besonders an der Jahresversammlung, die im Rahmen der Tagung der Schweizerischen Naturforschenden Gesellschaft (SNG) abgehalten wird.

Inscription auprès du secrétaire (Dr. med. Alfred W. Gubser, Medizinhistorisches Institut der Universität Zürich, Rämistrasse 71, 8006 Zürich) ou d'un autre membre du comité. Les membres reçoivent les Publications de la Société à un prix réduit. Les membres sont invités à assister à toutes les manifestations de la Société, en particulier à l'assemblée annuelle pendant la réunion annuelle de la Société Helvétique des Sciences Naturelles (SHSN).

\section{Manuskripte / Manuscrits}

Redaktion: Prof.Dr.med.Carl Haffter, Basel. Redaktionssekretariat: Frau Dr.phil. MarieLouise Portmann, Medizinhistorische Bibliothek, Klingelbergstraße 23, Ch-4031 Basel.

Die Beiträge müssen mit einem kurzen Résumé (in englischer Sprache) versehen sein. Fußnoten fortlaufend numerieren und an den Schluß stellen. Unterstrichenes wird kursiv gesetzt. Beiträge von über 20 Druckseiten erfordern einen Zuschuß des Autors. Autorkorrekturen und Illustrationen gehen zu Lasten der Verfasser.

Les manuscrits doivent être suivis d'un petit résumé en anglais. Nous prions de numéroter les notes progressivement et de les placer à la fin. Ce qui est souligné est mis en italique. Articles de plus de 20 pages imprimées nécessitent une participation financière de l'auteur. Les corrections de l'auteur et les illustrations devront être payées par les auteurs.

Vorstand/Comité: Präsident: Prof.Dr.med. Huldrych M.Koelbing, Zürich; Vizepräsident: Frau Prof. Dr. med. Esther Fischer-Homberger, Bern; Sekretär und Quästor: Dr. med.Alfred W.Gubser, Zürich; Redaktor des Gesnerus: Prof.Dr.med. Carl Haffter, Basel; Beisitzer: Prof.Dr.med. Hans H.Walser, Zürich, Prof.Dr.med. Jean Starobinski, Genève, Dr.med. dent. Roger Joris, Nyon.

Publiziert mit Unterstützung der Schweizerischen Naturforschenden Gesellschaft Publié avec l'aide de la Société Helvétique des Sciences Naturelles

Copyright (C) 1982 by Schweizerische Gesellschaft für Geschichte der Medizin und der Naturwissenschaften Member of CCG, Copyright Clearence Center, Inc., Salem, Massachusetts, USA 